EPJ Web of Conferences 41, 10007 (2013)

DOI: $10.1051 /$ epjconf/20134110007

(C) Owned by the authors, published by EDP Sciences, 2013

\title{
Coherent Electron Source for Ultrafast Electron Diffraction and Imaging
}

\author{
M. Müller ${ }^{1}$, A. Paarmann ${ }^{1}$, C. Xu ${ }^{1,2}$, and R. Ernstorfer ${ }^{1}$ \\ ${ }^{1}$ Fritz-Haber-Institut der Max-Planck-Gesellschaft, 14195 Berlin, Germany \\ ${ }^{2}$ Shanghai Institute of Optics and Fine Mechanics, Shanghai 201800, China
}

\begin{abstract}
We numerically investigate the suitability of photoexcited nanotips as a source of coherent femtosecond single electron pulses for ultrafast surface-sensitive electron diffraction and non-destructive imaging with low-energy electrons. The experimental parameters for realizing hundred femtosecond time resolution are identified by evaluating the effects of vacuum dispersion and beam divergence on the temporal broadening of the electron wave packet during its propagation to the sample.
\end{abstract}

\section{Introduction}

Within the last few years there has been tremendous progress in the generation of ultrashort electron pulses, which allow direct probing of femtosecond structural dynamics using optical pump-electron probe techniques such as ultrafast electron diffraction [1,2]. Moreover, the physics of photoemission from metal nanotips triggered by femtosecond laser pulses was recently investigated extensively [3-5]. Depending on the tip size, tip material, applied DC-voltage and laser intensity, various emission processes were observed and discussed in the literature. The emission area of such nanotips is confined to a few nanometers due to field enhancement, making them ideal candidates for an electron point source delivering highly coherent ultrashort electron pulses. Operated at low DCvoltages, nanotips are especially attractive as a pulsed low-energy electron gun for use in timeresolved electron diffraction experiments.

In view of this potential application and in particular for the time resolution, it is essential to consider propagation effects of the electron wave packets between tip and sample. First of all, space charge effects can be eliminated by using single electron pulses produced at a high repetition rate. The effect of vacuum dispersion is particularly crucial for low-energy electrons. In addition, geometric path length differences of the electrons due to the intrinsic beam divergence of the tip will cause further temporal broadening of the electron pulse. In order to investigate the dispersive and geometric contributions to the effective electron pulse duration, we model the properties of single electron wave packets by calculating the classical trajectories in the electric field of a nanometersized tip. Our results are applicable to pump-probe surface-sensitive electron diffraction in a reflective geometry as well as to ultrafast transmission experiments using forward diffraction and non-destructive imaging with low-energy electrons.

This is an Open Access article distributed under the terms of the Creative Commons Attribution License 2.0, which permits unrestricted use, distribution, and reproduction in any medium, provided the original work is properly cited. 


\section{Simulation Methods}

We calculate the electrostatic fields for the negatively biased nanotip placed above a grounded flat anode using a finite element software package (Comsol Multiphysics). Due to field enhancement, the maximum field strengths at the tip are in the range of $0.1-10 \mathrm{GV} / \mathrm{m}$. Assuming one electron per pulse, electron wave packet propagation is simulated classically by solving the equation of motion for single electrons in the inhomogeneous electric field of the tip using a Runge-Kutta algorithm. The initial electron properties are described by two-dimensional Gaussian distributions $F(E, \phi)$ of variable widths $\sigma_{E}$ for the initial kinetic energy $E$ and $\sigma_{\phi}$ for the emission angle $\phi$, respectively,

$$
F(E, \phi)=F_{0} \theta(E) \exp \left[-\frac{\left(E-E_{0}\right)^{2}}{2 \sigma_{E}^{2}}-\frac{\phi^{2}}{2 \sigma_{\phi}^{2}}\right] \text {. }
$$

Moreover, we assume electron emission normal to the tip surface, see Figure 1 (a). The flight time distribution $\tau(t)$, i.e. the probability of finding the electron at the anode after time $t$, is analyzed by evaluating the single trajectories weighted by their statistical probability according to $F(E, \phi)$. Figure 1 (b) shows a selection of distributions $\tau(t)$ for different values of $\sigma_{\phi}$ and $\sigma_{E}$, respectively. Depending on the specific values of $\sigma_{\phi}$ and $\sigma_{E}$, the flight time distribution can be highly asymmetric. The effective temporal pulse broadening at the anode is then analyzed by the standard deviation $\sigma_{\tau}$ of the distribution $\tau(t)$, which gives an upper limit for the FWHM of the electron pulse duration. Figure 1 (c) further shows the spatial broadening of the electron wave packet during its propagation.
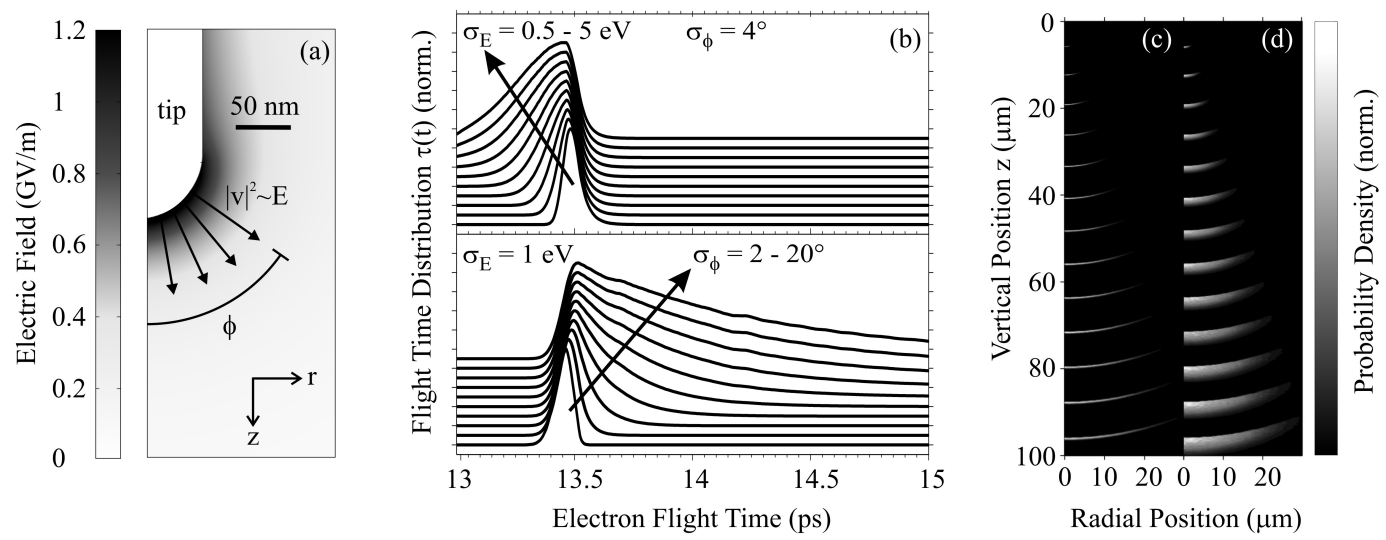

Fig. 1. (a) Simulation geometry with negatively biased tip and grounded anode. Electrons are emitted normal to the tip surface with angle $\phi$ and velocity $|v| \propto \sqrt{E}$. The scale shows the electric field for tip voltage $\mathrm{U}=-200 \mathrm{~V}$, tip radius $R=50 \mathrm{~nm}$ and tip-anode distance $D=100 \mu \mathrm{m}$. (b) Flight time distributions $\tau(t)$ of the electron wave packets for different initial energy widths $\sigma_{E}$ (top) and angular spreads $\sigma_{\phi}$ (bottom), both for mean initial energy of $E_{0}=0.5 \mathrm{eV}$. Simulation parameters are as in (a). Time series of spatial electron distributions in steps of 1 ps after emission for two energy widths $\sigma_{E}=0.5 \mathrm{eV}$ (c) and $\sigma_{E}=5 \mathrm{eV}$ (d), with $E_{0}=0.5 \mathrm{eV}$ and $\sigma_{\phi}=10^{\circ}$.

\section{Temporal Pulse Characteristics}

Our simulations show that the electron pulse duration at the anode is dominated by differences in the length of the trajectories between on- and off-axis electrons, and is dispersion controlled only for very small emission angles. In Figure 2 (a), we plot the standard deviation $\sigma_{\tau}$ as a function of $\sigma_{\phi}$ and $\sigma_{E}$ for a mean initial energy of $E_{0}=0.5 \mathrm{eV}$. For small angular spreads of $\sigma_{\phi}<4^{\circ}$, the horizontal lines indicate that vacuum dispersion is the main broadening mechanism. However, for larger beam 


\section{XVIIIth International Conference on Ultrafast Phenomena}

divergences and over a wide range of energy spreads $\sigma_{\mathrm{E}}$, the final pulse duration is dominated by path length differences. The transition between both regimes is illustrated in the line profiles for four different angular spreads $\sigma_{\phi}$.

The dependence of $\sigma_{T}$ on the tip voltage is shown in Figure 2 (b) plotted for different divergences $\sigma_{\phi}$. We see the expected decrease of the pulse duration with increasing tip voltage, i.e. larger final electron energy. Also here, we observe a transition from the dispersive to the path length controlled regime at $\sigma_{\phi} \sim 5^{\circ}$, above which the electron pulse duration scales as $\sigma_{T} \propto 1 / \sqrt{|U|}$, indicated by the linear behavior in the doubly logarithmic plotted line profiles on the right side of the contour plot.
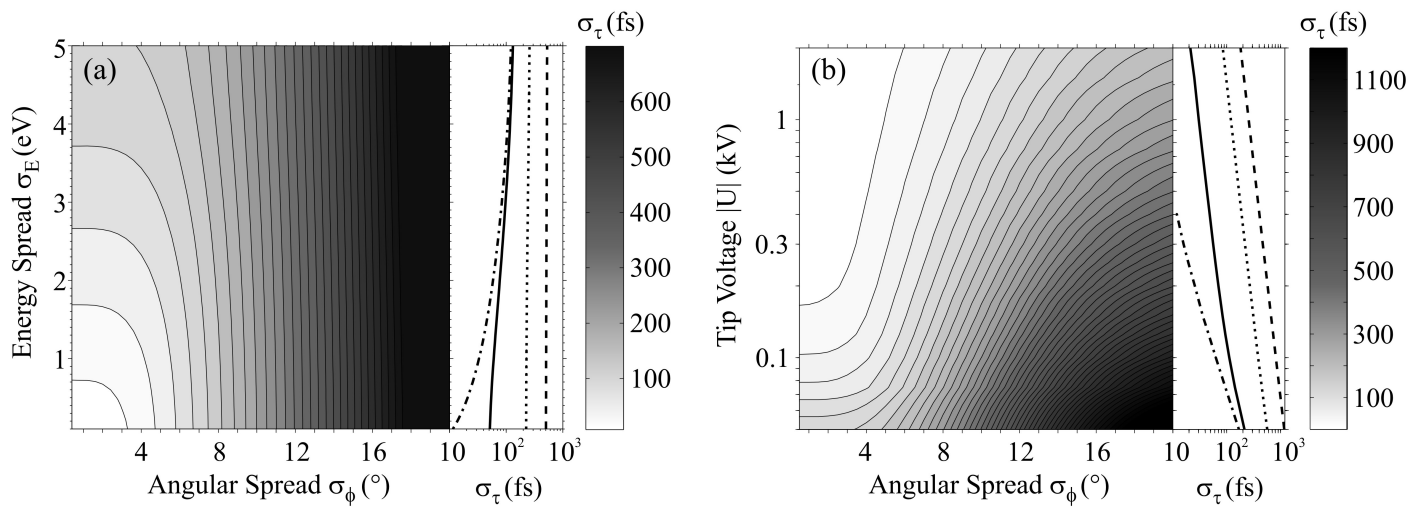

Fig. 2. Contour plots of the effective temporal pulse broadening $\sigma_{\tau}$ as a function of $\sigma_{\phi}$ and $\sigma_{E}$ for $U=-200 \mathrm{~V}$ (a), and of tip voltage $U$ for $\sigma_{E}=0.5 \mathrm{eV}$ (b). Contour lines are drawn every $25 \mathrm{fs}$. The simulation parameters are $E_{0}=0.5 \mathrm{eV}, R=50 \mathrm{~nm}$ and $D=100 \mu \mathrm{m}$. The line profiles on the right side of the contour plots were taken at $\sigma_{\phi}=2^{\circ}$ (dashed-dotted), $\sigma_{\phi}=5^{\circ}$ (solid), $\sigma_{\phi}=10^{\circ}$ (dotted) and $\sigma_{\phi}=15^{\circ}$ (dashed).

\section{Conclusions}

We investigated the effects of vacuum dispersion and beam divergence on the propagation of femtosecond single electron pulses excited from sharp metal tips. We analyzed the spatiotemporal properties of the electron wave packet during propagation depending on the initial energy distribution, emission angle, tip voltage, tip radius, and tip-anode distance. In terms of temporal pulse broadening, the results demonstrate the importance of path length differences compared to vacuum dispersion in the highly inhomogeneous field of the nanotip. Minimizing the beam divergence, e.g. by implementing compact electron optics, will enable the realization of tip-based ultrafast low-energy electron diffraction and imaging with $100 \mathrm{fs}$ time resolution.

1. G. Sciaini and R.J.D. Miller, "Femtosecond electron diffraction: heralding the era of atomically resolved dynamics", Rep. Prog. Phys.74, 096101 (2011)

2. H. Ihee, V.A. Lobastov, U.M. Gomez, B.M. Goodson, R. Srinivasan, C.Y. Ruan and A.H. Zewail, "Direct imaging of transient molecular structures with ultrafast diffraction", Science 291, 458-62 (2001)

3. G Herink, D.R. Solli, M. Gulde, and C. Ropers, "Field-driven photoemission from nanostructures quenches the quiver motion“, Nature 483, 190 (2012)

4. M. Krüger, M. Schenk and P. Hommelhoff, "Attosecond control of electrons emitted from a nanoscale metal tip", Nature 475, 78 (2011)

5. H. Yanagisawa, M. Hengsberger, D. Leuenberger, M. Klöckner, C. Hafner, T. Greber and J. Osterwalder, "Energy Distribution Curves of Ultrafast Laser-Induced Field Emission and Their Implications for Electron Dynamics", Phys. Rev. Lett. 107, 087601 (2011) 